\title{
Electrophysiologic Changes following Treatment with Organophosphorus- Induced Delayed Neuropathy-Producing Agents in the Adult Hen
}

\author{
Donald G. Robertson, ${ }^{*} \uparrow$ BRadley W. SChWab, ${ }^{*}$ Robert D. Sills, ${ }^{*}$ \\ RUDY J. RICHARDSON, ${ }^{*}$ AND REBECCA J. ANDERSON*‡ \\ *Toxicology Program, School of Public Health. The University of Michigan, Ann Arbor, Michigan 48105; \\ $\dagger$ Department of Pathology and Experimental Toxicology. Warner-Lambert/Parke-Davis Pharmaceutical Research \\ Ann Arbor, Michigan 48105; and $\ddagger$ Department of Pharmacology, Warner-Lambert/Parke-Davis \\ Pharmaceutical Research, Ann Arbor, Michigan 48105
}

Received July 7, 1986; accepted November 3, 1986

\begin{abstract}
Electrophysiologic Changes following Treatment with Organophosphorus-Induced Delayed Neuropathy-Producing Agents in the Adult Hen. RoBerTSON, D. G., SCHWAB, B. W., Sills, R. D., Richardson, R. J., ANd Anderson, R. J. (1987). Toxicol. Appl. Pharmacol. 87, 420429. Although clinical, pathological, and biochemical effects of organophosphorus-induced delayed neuropathy (OPIDN) have been intensively investigated in the adult hen, detailed electrophysiological studies are lacking. Adult white leghorn hens were treated with a single oral dose of either $30 \mathrm{mg} / \mathrm{kg}$ tri-2-cresyl phosphate (TOCP), $750 \mathrm{mg} / \mathrm{kg}$ TOCP, $4 \mathrm{mg} / \mathrm{kg}$ di- $n$-butyl-2,2dichlorovinyl phosphate (DBCV), or $30 \mathrm{mg} / \mathrm{kg}$ di-n-butyl-2,2-dichlorovinyl phosphinate (DBCV-P). The $750 \mathrm{mg} / \mathrm{kg}$ TOCP and DBCV, but not the $30 \mathrm{mg} / \mathrm{kg}$ TOCP and DBCV-P. treatments resulted in clinical signs of OPIDN and mild to marked damage of the tibial nerve 21 days after dose. Twenty-four hr lymphocyte neurotoxic esterase (NTE) inhibition was used as an index of brain NTE inhibition for the various organophosphorus compound (OP) treatment. Twenty-four hr lymphocyte NTE inhibition for $30 \mathrm{mg} / \mathrm{kg}$ TOCP, $750 \mathrm{mg} / \mathrm{kg}$ TOCP, DBCV, and DBCV-P was $54.1,87.1,84.8$, and $68.3 \%$. respectively. Twenty-one days after dose, the TOCP-treated hens exhibited some abnormalities in conduction velocity and action potential duration in the tibial or sciatic nerves. No abnormalities were observed in action potential parameters of either the DBCV or DBCV-P treatments. Neurotoxic OP (TOCP and DBCV) treatment resulted in decreased refractoriness in the tibial nerve, increased refractoriness in the sciatic nerve, and elevated strength duration threshold for both nerves. These changes were not present in nerves from DBCV-P (a non-neurotoxic NTE inhibitor)-treated hens. These results suggest that refractory period and strength duration abnormalities in peripheral nerve correlate well with the production of OPIDN and are evident without coincident clinical signs or histopathology. (c) 1987 Academic Press, lnc.
\end{abstract}

Organophosphorus-induced delayed neuropathy (OPIDN) is a well-characterized clinical syndrome that has been under investigation for over 50 years. This syndrome is best defined as a sensorimotor axonopathy which particularly affects long, large diameter axons in a distal-to-proximal fashion (dying-back neuropathy). Structure-activity relationships of organophosphorus compounds (OPs) producing OPIDN have been well documented and are the subject of several reviews (John- son, 1975; Davis and Richardson, 1980; Abou-Donia, 1981; Davis et al., 1985). The capacity of OPs to produce OPIDN correlates with their ability to inhibit neurotoxic esterase (NTE), although there are compounds that inhibit NTE that are not neurotoxic. Johnson (1974) has suggested that the necessary step for the production of OPIDN is the cleavage of a phosphoryl ester bond to leave a charged monosubstituted phosphoric acid group on the enzyme, a process called aging. 
Although the relationship between NTE organophosphorylation and OPIDN production has been rigorously investigated, a mechanistic link between these two actions has remained elusive.

One problem with mechanistic investigations of OP neuropathy is that typically hen brain is used to assess NTE inhibition $24 \mathrm{hr}$ after dosing, even though clinical and histopathological signs of OPIDN do not usually become apparent for 8-21 days. Recovery of NTE activity after inhibition by OPs is fairly rapid with a $t \frac{1}{2}$ for recovery ranging from 4 to 7 days (Johnson, 1975). Therefore, NTE activity approaches control values at the onset of peripheral neuropathy. Most previous studies of NTE and OPIDN have used paired groups of animals: one for brain NTE activity measurements and the other for measurement of some aspect of OPIDN. However, because of the individual variation in the degree of inhibition of NTE and neuropathic susceptibility following a given dose of $\mathrm{OP}$, correlations between these two effects have been further confounded. Our work (Dudek and Richardson, 1980, 1982; Richardson and Dudek, 1983) has demonstrated significant NTE activity in hen peripheral lymphocytes, and we have shown that brain and lymphocyte NTE activities are well correlated within $24 \mathrm{hr}$ after single doses of neurotoxic OPs (Schwab and Richardson, 1986). Therefore by using lymphocytes to measure NTE, assessment of OPIDN and NTE can be obtained from the same animal, eliminating one key source of variation.

Electrophysiological assessment of peripheral nerves after treatment with OP compounds has primarily been studied in the cat (Lowndes et al., 1974; Baker and Lowndes, 1980; Baker et al., 1980), the rat (Averbook and Anderson, 1983; Anderson, 1983; Anderson and Dunham, 1985), and to a limited extent in the hen (Durham and Ecobichon, 1984). These studies focused largely on measurements of conduction velocity, which is largely unchanged following OP administration. Occasionally other functional measurements (relative refractory period and sensory afferent activity) have been made, but no previous studies have systematically examined the effect of OPs on a variety of electrophysiologic characteristics in the hen. The purpose of this study was to examine a variety of electrophysiological parameters in the hen at a time when the animal was expressing overt clinical signs of OPIDN. Peripheral lymphocyte NTE activity was assessed $24 \mathrm{hr}$ after dosing. Tri-2-cresyl phosphate (TOCP), which requires metabolic activation to become neurotoxic, and di- $n$-butyl-2,2-dichlorovinyl phosphate (DBCV) were chosen as model OPIDN compounds. In addition, the phosphinate analog of DBCV, di- $n$-butyl-2,2dichlorovinyl phosphinate (DBCV-P, a nonneurotoxic NTE inhibitor), was used to control for the effects of NTE inhibition on peripheral nerve electrical activity.

\section{METHODS}

Animals and housing. Adult ( $>1$ year old, 1.4-2.2 kg) white leghorn hens (Omega Chicks, Haslett, MI) were maintained in groups of three to four in stainless-steel cages $(0.8 \times 0.8 \times 0.9 \mathrm{~m})$ with wire-mesh floors in a temperature-controlled $\left(21-23^{\circ} \mathrm{C}\right)$ room with a 12 -hr light/12hr dark cycle. Except for predose fasting, food (Purina Layena Mash) and tap water were available ad libitum.

Dosing. All OPs were dissolved in Mazola corn oil and administered as a single dose in a volume of $2 \mathrm{ml} / \mathrm{kg}$ by oral cannula directly into the crop of hens fasted for 24 $\mathrm{hr}$. Two groups of hens received 30 and $750 \mathrm{mg} / \mathrm{kg}$ TOCP (practical grade, Sigma Chemical Co., St. Louis, MO), respectively. Additional groups of hens were dosed with either $4 \mathrm{mg} / \mathrm{kg}$ DBCV or $30 \mathrm{mg} / \mathrm{kg}$ DBCV-P, while the control group received vehicle alone. $\mathrm{DBCV}$ and $\mathrm{DBCV}$ $P$ were custom-synthesized by Ash Stevens Inc. (Detroit, MI) and the purity of these compounds was determined to be $\geqq 99 \%$ by gas chromatography-mass spectrometry. A minimum of seven hirds was dosed in each group. The higher TOCP dose and the DBCV dose were chosen on the basis of preliminary studies which demonstrated marked NTE inhibition for both treatments as well as the production of clinical signs of OPIDN within 21 days. The lower dose of TOCP produced approximately $50 \%$ NTE inhibition and no clinical signs in preliminary studies. The dose of DBCV-P was selected in preliminary studies to be equipotent with the neurotoxic DBCV for NTE inhibition. Hens were weighed prior to and 21 days after dose and were observed daily for clinical signs of toxicity. Clinical evidence of OPIDN was graded as follows: $0=$ no signs, $1=$ gait abnormalities, $2=$ animal 
observed resting on haunches but the ability to stand still remains, and 3 = complete prostration.

NTE assays. Blood (7-10 ml) was taken from the wing vein using a heparinized syringe $24 \mathrm{hr}$ after dosing. Lymphocytes were isolated and NTE assays were conducted as described by Schwab and Richardson (1986). Protein was determined using a Bio-Rad protein assay kit (BioRad Laboratories, Richmond, $\mathrm{CA}$ ) using bovine serum albumin, fraction V (Sigma Chemical, St. Louis, MO) as a standard. Specific activity was then calculated as $\mathrm{nmol}$ suhstrate hydrolyzed $\mathrm{min}^{-1} \mathrm{mg}$ protein ${ }^{-1}$

Lymphocyte NTE activity can be used as an index of brain NTE inhibition for TOCP and DBCV (Schwab and Richardson, 1986). However, a correlation between lymphocyte and brain NTE inhibition after DBCV-P administration was not available. Therefore, prior to these studies, a dose-response curve for DBCV-P was generated for both lymphocyte and brain NTE (24 hr after dosing) and the correlation was established using the method described by Schwab and Richardson (1986).

Electrophysiology. Birds were examined for electrophysiological changes 2 I days after dosing. Surgical and electrophysiological methods have been described elsewhere (Robertson and Anderson, 1986). Briefly, hens were anesthetized with $60 \mathrm{mg} / \mathrm{kg} \alpha$-chlorolose (Fisher Scientific, Detroit, MI) which was injected into a wing vein. Both the tibial and sciatic nerves were surgically isolated. A short segment $(2-4 \mathrm{~mm})$ of the distal end of the tibial nerve was removed and placed in $6 \%$ phosphate (Sorenson's, pH 7.4)-buffered glutaraldehyde for fixation and later histology. During recording, nerves were immersed in mineral oil maintained at $39.5 \pm 0.1^{\circ} \mathrm{C}$ by means of a heat lamp coupled to a Thermistemp temperature controller (Yellow Springs Instruments, Yellow Springs, $\mathrm{OH}$ ). The activities from both the tibial and the sciatic nerves were recorded in a similar manner. Nerves were positioned on pairs of bipolar stimulating and recording electrodes fixed in a plastic holder maintaining the interelectrode distance at $30 \mathrm{~mm}$. A computer-assisted method for recording and analyzing the nerve compound action potential has been described previously (Anderson et al., 1981). Briefly, 50 supramaximal stimuli of $0.02-\mathrm{msec}$ duration were presented at 15 sec intervals. The following characteristics were obtained for each compound action potential: (1) onset conduction velocity, (2) peak conduction velocity, (3) action potential duration, and (4) action potential amplitude. After the characteristics of the action potential were obtained, the relative refractory period for the nerve was measured. This was done using twin pulse stimulation at interstimulus intervals from 1 to $8 \mathrm{msec}$. The relative refractory period was calculated using a modification of the method of Roberts and Trollope (1979) and converted to linear form using the method of Anderson (1983).

Strength-duration curves were obtained by measuring the voltage required to elicit threshold depolarization of the nerve at stimulus durations from 10 to $1000 \mu \mathrm{sec}$.
Chronaxie and rheobase measurements were obtained from these curves. In addition, the inflexion region of the curve $(40-100 \mu \mathrm{sec})$ was converted to a linear form by logarithmic transformation of both the ordinate and abscissa to facilitatc comparison of the treatment groups.

Histology: The tibial nerve segments removed before recording were allowed to fix for $3 \mathrm{hr}$, washed in Soren-

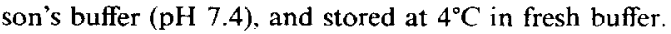
Nerves were eventually dehydrated through a graded series of ethanol solutions and embedded in Spurr resin (Polysciences, Warrington, PA), placed in flat molds, and hardened in an oven at $60^{\circ} \mathrm{C}$. Sections $(1 \mu \mathrm{m})$ were cut on an ultramicrotome, stained with toluidine blue, and observed for histological changes by light microscopy.

Statistics. Analysis of variance with the NewmanKeuls method of multiple comparisons was used to determine statistical difference between mean compound action potential data with $p<0.05$ set as the criterion of significance. The linear data (relative refractory period and strength-duration curves) were compared using analysis of covariance to determine significant differences in both slope and intercept of calculated lines. All data are expressed as means \pm standard error.

\section{RESULTS}

Both the $750 \mathrm{mg} / \mathrm{kg}$ TOCP- and DBCVtreated birds demonstrated clinical signs of toxicity as early as 12 days after dosing. These signs included gait abnormalities which became progressively more severe and in some cases led to complete ataxia. The $750 \mathrm{mg} / \mathrm{kg}$ TOCP-dosed birds appeared to be affected to a greater extent than the DBCV-treated birds. DBCV birds, although exhibiting obvious gait impairment, nevertheless appeared to be in good general health and did not lose as much weight as the TOCP-treated birds. The lower dose of TOCP did not elicit any sign of gait abnormalities. However, the animals were noticeably unkempt and lost weight. No clinical signs of toxicity were observed in the DBCV-P-treated group. A summary of clinical signs and weight changes during the timecourse of the experiment is presented in $\mathrm{Ta}$ ble 1 .

Although no animals died during the study, a number of treated birds did not survive the anesthesia or surgery. This resulted in different numbers of birds for the various experiments. Except where noted, at least five hirds were tested for each experiment. 
TABLE 1

INDICES OF TOXICITY TO HENS EXPOSED TO VARIOUS OP TREATMENTS

\begin{tabular}{|c|c|c|c|c|c|c|}
\hline \multirow[b]{2}{*}{ Treatment $(N)^{d}$} & \multirow{2}{*}{$\begin{array}{l}\text { Weight change }{ }^{a} \\
\text { (g) }\end{array}$} & \multicolumn{4}{|c|}{$\begin{array}{c}\text { Clinical signs }^{h} \\
(\%)\end{array}$} & \multirow{2}{*}{$\begin{array}{c}\text { Lymphocyte NTE" } \\
\text { inhibition } \\
(\%)\end{array}$} \\
\hline & & 0 & 1 & ? & 3 & \\
\hline Control (12) & $+88 \pm 38$ & 100 & 0 & 0 & 0 & - \\
\hline $30 \mathrm{mg} / \mathrm{kg}$ TOCP $(7)$ & $-120 \pm 48$ & 100 & 0 & 0 & 0 & $54.1 \pm 8.3$ \\
\hline $750 \mathrm{mg} / \mathrm{kg}$ TOCP (8) & $-348 \pm 71$ & 0 & 25 & 37.5 & 37.5 & $87.1 \pm 2.0$ \\
\hline $4 \mathrm{mg} / \mathrm{kg}$ DBCV $(7)$ & $-62 \pm 113$ & 0 & 71.5 & 28.5 & 0 & $84.8 \pm 2.8$ \\
\hline $30 \mathrm{mg} / \mathrm{kg} \mathrm{DBCV}-\mathrm{P}(8)$ & $+104 \pm 32$ & 100 & 0 & 0 & 0 & $68.3 \pm 6.9$ \\
\hline
\end{tabular}

${ }^{a}$ Average weight of animals at sacrifice - average predose weight.

${ }^{h}$ Clinical signs of neurotoxicity at sacrifice (\% of animals in group): $0=$ no signs; $1=$ gait abnormalities; $2=$ animal rests on haunches but can stand; and $3=$ completely ataxic.

( 1 - Mean NTE activity of treated group/mean NTE activity of control animals) $\times 100$.

"Number of animals in each group.

The results of the study investigating the correlation of hen brain and lymphocyte NTE inhibition by DBCV-P is presented in Fig. 1. The correlation was statistically significant $\left(r^{2}=0.57, p<0.01\right)$ and the higher end of the dose-response curve $(>20 \mathrm{mg} / \mathrm{kg}$ ) demonstrated particularly good agreement.

The results of the 24-hr lymphocyte NTE assays in the various treatment groups are

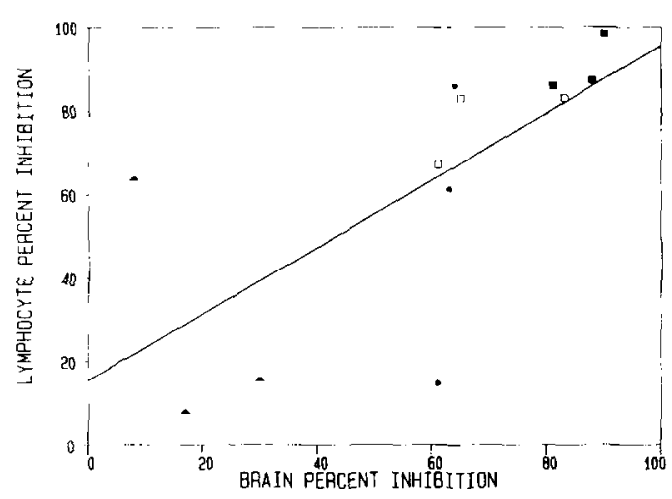

FIG. 1. Relationship between inhibition of lymphocyte NTE and inhibition of brain NTE in hens treated with either 2 (4), 5 (ச), 20 (C), or $30(\mathbf{C}) \mathrm{mg} / \mathrm{kg}$ DBCV-P $24 \mathrm{hr}$ after dosing. Each point represents an individual animal. The coefficient of determination $\left(r^{2}\right)$ for the line is 0.57 $(p<0.01, t$ test). Control lymphocyte NTE activity was $5.96 \pm 1.83 \mathrm{nmol}$ phenyl valerate hydrolyzed $/ \mathrm{min} \times \mathrm{mg}$ protein $(n=8)$. Control brain NTE activity was 24.37 $\pm 3.20 \mathrm{nmol}$ phenyl valerate hydrolyzed $/ \mathrm{min} \times \mathrm{mg}$ protein $(n=8)$. presented in Table 1. Only four hens were assayed for the DBCV assay. All birds in the high TOCP and DBCV groups had lymphocyte NTE inhibitions above $70 \%$ and all displayed OPIDN. Interestingly, two birds in the low TOCP group also had lymphocyte NTE inhibition above $70 \%$ (71 and $82 \%$ ), yet neither displayed any motor impairment. Although preliminary studies indicated that the $30 \mathrm{mg} / \mathrm{kg}$ dose of DBCV-P should have inhibited lymphocyte NTE by greater than $80 \%$, the mean value was only $68 \%$. Three out of the seven treated animals had lymphocyte NTE inhibitions greater than $70 \%$.

Table 2 presents the compound action potential parameter measurements obtained for the various treatment regimens for both the tibial and sciatic nerves. Both TOCP treatments resulted in significant changes in action potential parameters, whereas neither DBCV or DBCV-P treatment produced any significant difference in the action potential. Action potential duration was significantly increased in the tibial nerve, and onset conduction velocity was significantly decreased in the sciatic nerve with TOCP.

The relative refractory periods of both the tibial and sciatic nerves were significantly altered by all three neurotoxic OP treatments. The sciatic nerve refractory period was shifted to the right by neurotoxic OP treat- 
TABLE 2

Compound action Potential Parameters for Tibial and Sciatic Nerve of Control and Treated Hens

\begin{tabular}{|c|c|c|c|c|c|c|}
\hline Treatment & $\begin{array}{c}\text { Onset } \\
\text { conduction } \\
\text { velocity } \\
(\mathrm{m} / \mathrm{sec})\end{array}$ & $\begin{array}{c}\text { Peak } \\
\text { conduction } \\
\text { velocity } \\
(\mathrm{m} / \mathrm{sec})\end{array}$ & $\begin{array}{c}\text { Amplitude } \\
\text { (mV) }\end{array}$ & $\begin{array}{l}\text { Duration } \\
\text { (msec) }\end{array}$ & $\begin{array}{c}\text { Chronaxie } \\
\text { (msec) }\end{array}$ & $\begin{array}{l}\text { Rheobase } \\
(\mathrm{mV})\end{array}$ \\
\hline \multicolumn{7}{|l|}{ Tibial nerve } \\
\hline Control & $41.3 \pm 2.8$ & $26.5 \pm 1.6$ & $0.67 \pm 0.12$ & $1.08 \pm 0.11$ & $0.019 \pm 0.001$ & $1.70 \pm 0.06$ \\
\hline $30 \mathrm{mg} / \mathrm{kg}$ TOCP & $35.3 \pm 1.9$ & $22.0 \pm 1.0$ & $0.30 \pm 0.09$ & $1.69 \pm 0.30^{a}$ & $0.020 \pm 0.001$ & $1.84 \pm 0.08$ \\
\hline $750 \mathrm{mg} / \mathrm{kg}$ TOCP & $39.2 \pm 2.7$ & $21.7 \pm 1.5$ & $0.24 \pm 0.05$ & $1.76 \pm 0.23^{a}$ & $0.023 \pm 0.002$ & $1.97 \pm 0.11$ \\
\hline $4 \mathrm{mg} / \mathrm{kg} \mathrm{DBCV}$ & $41.8 \pm 2.5$ & $28.3 \pm 1.3$ & $0.66 \pm 0.13$ & $0.96 \pm 0.08$ & $0.026 \pm 0.001^{a}$ & $1.91 \pm 0.10$ \\
\hline $30 \mathrm{mg} / \mathrm{kg} \mathrm{DBCV}-\mathrm{P}$ & $40.1 \pm 2.8$ & $27.6 \pm 1.9$ & $1.06 \pm 0.29$ & $1.07 \pm 0.15$ & $0.024 \pm 0.001$ & $1.60 \pm 0.11$ \\
\hline \multicolumn{7}{|l|}{ Sciatic nerve } \\
\hline Control & $60.5 \pm 3.0$ & $32.0 \pm 2.2$ & $1.10 \pm 0.17$ & $1.21 \pm 0.06$ & $0.024 \pm 0.001$ & $1.80 \pm 0.03$ \\
\hline $30 \mathrm{mg} / \mathrm{kg}$ TOCP & $48.3 \pm 4.1^{a}$ & $27.0 \pm 1.3$ & $1.04 \pm 0.35$ & $1.68 \pm 0.05$ & $0.027 \pm 0.001$ & $1.90 \pm 0.07$ \\
\hline $750 \mathrm{mg} / \mathrm{kg}$ TOCP & $43.1 \pm 1.9^{a}$ & $25.6 \pm 1.6$ & $0.78 \pm 0.16$ & $1.30 \pm 0.07$ & $0.028 \pm 0.002$ & $1.97 \pm 0.08$ \\
\hline $4 \mathrm{mg} / \mathrm{kg} \mathrm{DBCV}$ & $57.6 \pm 1.9$ & $30.6 \pm 1.4$ & $1.30 \pm 0.20$ & $1.17 \pm 0.10$ & $0.035 \pm 0.001^{a}$ & $2.01 \pm 0.11$ \\
\hline $30 \mathrm{mg} / \mathrm{kg} \mathrm{DBCV}-\mathrm{P}$ & $54.4 \pm 1.8$ & $31.5 \pm 1.9$ & $1.45 \pm 0.36$ & $1.05 \pm 0.04$ & $0.038 \pm 0.003^{a}$ & $1.55 \pm 0.04^{a}$ \\
\hline
\end{tabular}

${ }^{a} p<0.05$.

ment (Fig. 2a) while the tibial nerve was shifted to the left (Fig. 2b). In addition, there was no evidence of supranormal response observed in the oscilloscope traces of either nerve. There was a trend toward a decreased slope in the relative refractory period of the sciatic nerve from OP-treated birds. However, this effect was not statistically significant. No change in slope was observed for the tibial nerve. DBCV-P did not significantly alter the relative refractory period for either nerve.
The strength-duration curves for both nerves showed an increase in threshold excitability of the nerves following neurotoxic $\mathrm{OP}$ treatment (Fig. 3). This increase manifested itself as a parallel upward shift in the strength-duration curve. The shift due to TOCP treatment was dose dependent in both nerves. DBCV treatment was more effective in inducing these changes than was either dose of TOCP. No change was observed in animals treated with DBCV-P. Chronaxie and rheobase calculations are presented in
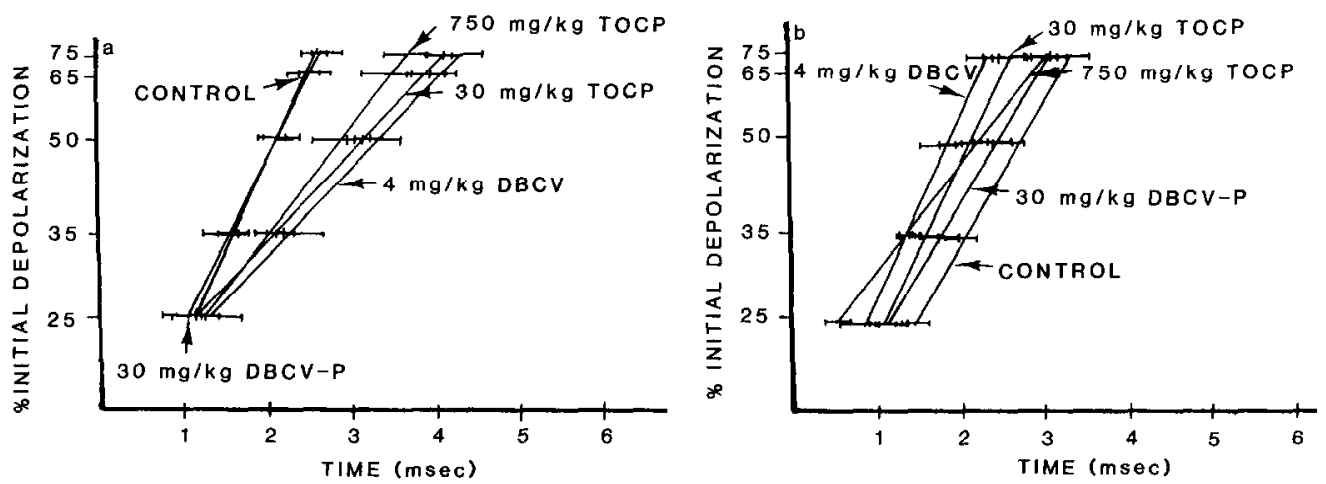

FIG. 2. Log-linear representations of the relative refractory period for sciatic (a) and tibial (b) nerves after various OP treatments. Refractory period shifts were statistically significant (analysis of covariance, $p<0.05$ ) for all neurotoxic OP treatments (TOCP and DBCV) but not for DBCV-P. 

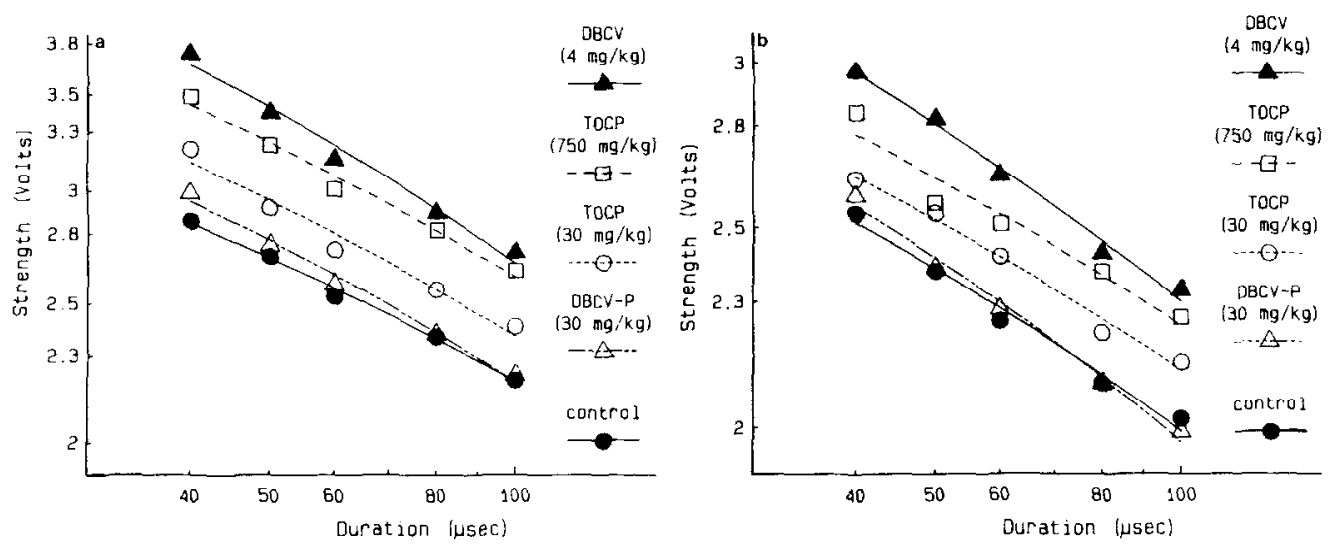

FIG. 3. Log-log representation of the inflection region (40-100 $\mu \mathrm{sec})$ of strength-duration curves from sciatic (a) and tibial (b) nerves of hens after various OP treatments. The strength duration threshold was significantly elevated (analysis of covariance, $p<0.05$ ) for all neurotoxic OP treatments but not for DBCV-P.

Table 2. OP treatment resulted in increases of both chronaxie and rheobase measurements. However, the only statistically significant change noted in the tibial nerve was a slight increase in chronaxie after DBCV treatment. In the sciatic nerve DBCV treatment resulted in a significant increase in chronaxie. In addition the chronaxie of DBCV-P-treated hens was significantly elevated while the rheobase was significantly decreased.

Microscopic investigation of tibial nerves revealed evidence of axonal damage only in the DBCV and $750 \mathrm{mg} / \mathrm{kg}$ TOCP groups. There was wide variation in individual animal responses within these two groups. TOCP treatment resulted in various pathological sequelae including swollen axons, dark staining axoplasm, disrupted myelin, phagocyte infiltration, and complete axonal degeneration (Fig. 4b). Occasionally, phagocytes were observed containing axonal debris. These effects were also noted in tibial nerves from DBCV-treated birds; however, axonal damage was not as extensive as that noted with TOCP treatment. Control specimens demonstrated mild myelin disruption (Fig. 4a). However, no other evidence of abnormal morphology was present.

\section{DISCUSSION}

Doses of neurotoxic OPs sufficient to cause OPIDN within 21 days also caused pro- nounced changes in two electrophysiological parameters, the relative refractory period, and the strength-duration curve. These changes were not related to NTE inhibition alone, since DBCV-P (a nonageable and therefore nonneurotoxic NTE inhibitor) did not produce any of the changes seen after administration of the neurotoxic OPs. In addition, the neurotoxic OPs induced electrophysiologic changes in animals given a dose of TOCP that did not produce any clinical or histopathological signs of OPIDN.

The neurotoxic OPs had a pronounced effect on the relative refractory period. Sciatic nerves became more refractory, indicating that their ability to carry high frequency impulses was impaired. Treatment with DBCV$P$ (a nonaging NTE inhibitor) had no effect on this measurement, suggesting that the observed disruption of nerve impulse conduction correlates with the ability of an OP to age. It is important to note that the low dose of TOCP, producing only $54 \%$ lymphocyte NTE inhibition and no clinical signs of toxicity, was just as effective at altering the relative refractory period as the higher TOCP and DBCV treatments. The refractory period of the tibial nerve was shifted to the left suggesting that the nerve actually became less refractory after OP treatment. Although there are other examples of compounds that shift the 

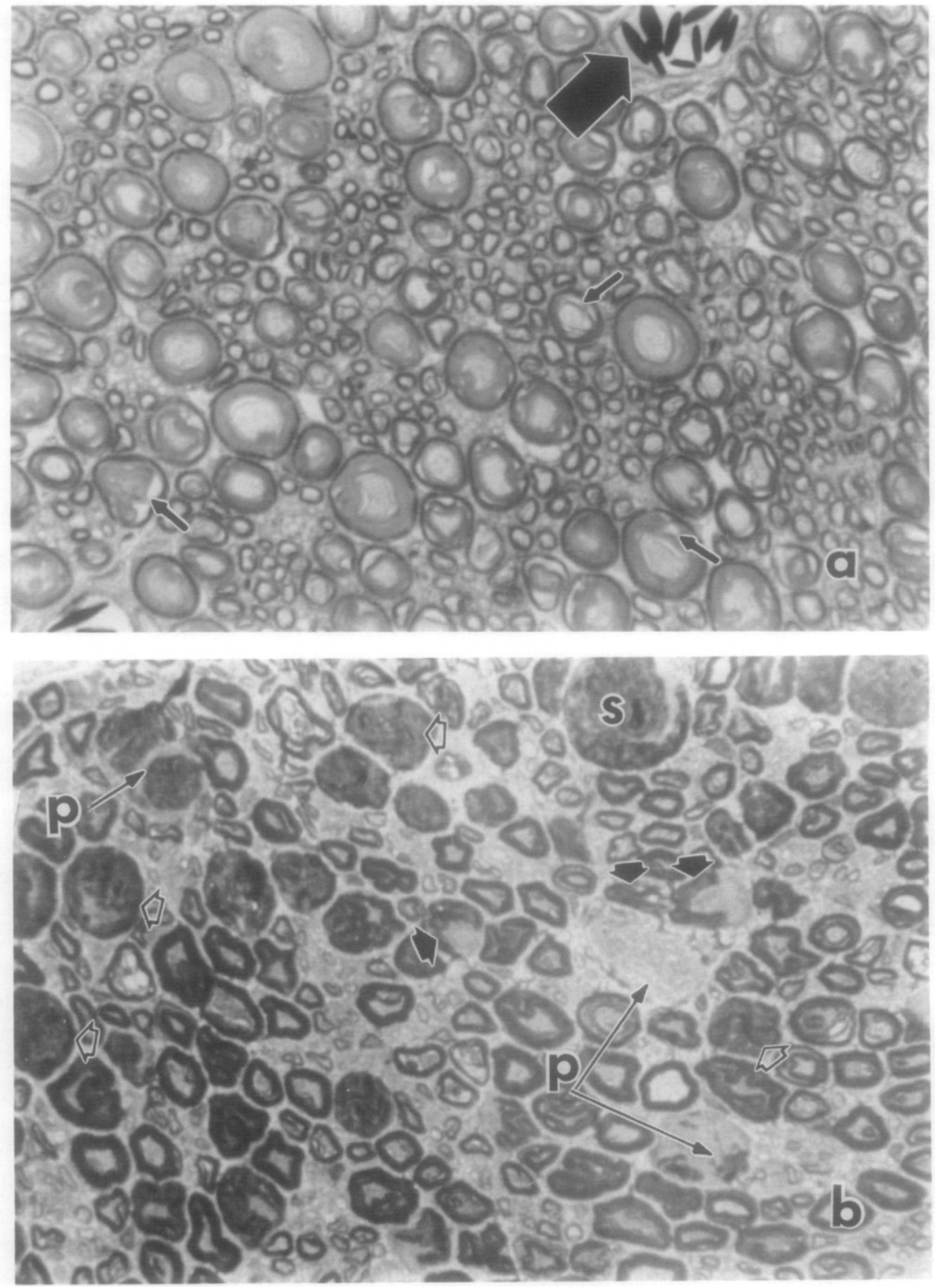

FIG. 4. Micrographs of $1-\mu \mathrm{m}$ tibial nerve cross sections. (a) Control nerve showing artifactual myelin disruption (small arrows) and the presence of a blood vessel (large arrow). (b) Nerve from hen treated with $750 \mathrm{mg} / \mathrm{kg}$ TOCP. Note the grossly swollen axon (s), numerous axons with darkly staining axoplasm (open arrow heads), phagocytes (p), and totally degenerated axons (solid arrow heads) which may or may not be contained within phagocytic cells. Toluidine blue stain, $800 \times$ original magnification. 
relative refractory period to the left (Averbook and Anderson, 1983), it is difficult to reconcile the effect of the neurotoxic OPs which shift the refractory period of the sciatic nerve to the right and the tibial nerve to the left. It should be noted that activity from the tibial nerve was recorded more distally than from the sciatic nerve. Since all measurements were made at 21 days after treatment and these OPs are known to produce a progressive distal-to-proximal neuropathy, the condition of the more distal tibial nerve would not be expected to be at the same state of OPIDN as the more proximal sciatic nerve.

The other electrophysiological parameter that was significantly affected by OP treatment was the strength-duration curve. Clinical neuroscientists have used this technique for evaluating a number of conditions including diabetic-, uremic, and alcohol-induced peripheral neuropathies (Friedli and Meyer, 1984). However, this measurement has seldom been used in studies of organophosphorus compounds. Neurotoxic OP treatment resulted in significant changes in both tibial and sciatic nerves. These changes, as with the refractory period abnormalities, were related to those compounds which produce NTE inhibition and also age. Observed elevations in the strength-duration curves suggest that neurotoxic OP treatment alters axonal membrane properties, resulting in elevated thresholds of peripheral nerves. An important observation was that this effect occurred in the low dose TOCP group which showed no clinical evidence of OPIDN. The degree of threshold elevation following TOCP appeared to be dose related. However, in individual animals, the increase in the strength-duration curves did not correlate well with lymphocyte NTE inhibition. Along with the elevation of strength-duration curves there was also an increased chronaxie noted for all DBCVtreated tibial and sciatic nerves. Chronaxie is an index of nerve responsiveness calculated from the strength-duration curve and it permits comparison between treatment groups. The increase in chronaxie represents an increased threshold for nerve conduction in the
OP-treated nerves. (It should be noted that the increased chronaxie noted with DBCV-P in the sciatic nerve was the result of a significant decrease in the rheobase.)

Both TOCP-treated groups displayed significant action potential disruption in both the tibial and sciatic nerves (Table 2). These abnormalities were characterized by decreased onset conduction velocity in the sciatic nerve and increased action potential duration in the tibial nerve. Despite the pronounced effects of TOCP on both sciatic and tibial action potential, DBCV had no effect on any action potential parameter. This suggests that the changes observed with TOCP were not related to NTE inhibition or the production of OPIDN. These electrophysiologic results, along with the weight loss and unkempt appearance observed in the TOCP. treated hens, suggested that the action potential abnormalities following TOCP treatment may be secondary to other toxic actions of the compound. Durham and Ecobichon (1984) reported no change in sciatic nerve conduction velocity in hens given single oral doses of $500 \mathrm{mg} / \mathrm{kg}$ TOCP and sacrificed at 7, 14, 28, 42, and 56 days after treatment.

Perhaps the difference between our study and that of Durham and Ecobichon was the purity of the TOCP. Practical grade TOCP frequently has various impurities which may vary from batch to batch. These impurities may be responsible for variations in toxicity (Gary Sprague, Personal communication). The results obtained using low potency OPs in experimental OPIDN studies should therefore be interpreted with caution.

Lymphocyte NTE inhibition proved to be a reliable indicator of hrain NTE inhibition. In addition, the correlation of DBCV-P-induced inhibition of brain with inhibition of lymphocyte NTE suggests that DBCV and DBCV-P make an excellent pair of structural analogs for studying the relationship of NTE inhibition and the production of OPIDN. It has been recognized for some time that a necessary fraction of NTE must be inhibited before clinical signs of OPIDN are manifest. This fraction has generally been accepted 
as approximately $70 \%$ (Johnson, 1975). Whether this implies an actual threshold or not is currently the subject of some debate (Schwab and Richardson, 1986).

The morphology of the tibial nerve demonstrated conclusive evidence of OPIDN. The milder lesions noted in nerves from $\mathrm{DBCV}$ treated hens as compared to $750 \mathrm{mg} / \mathrm{kg}$ TOCP-treated hens correlated with the less severe clinical signs noted in the former group. The structural damage described was typical of that noted for OPIDN, as recently described for the fibular nerve of hens exposed to DFP (disopropyl fluorophosphate) (Ehrich et al., 1985). Since electrophysiologic assessment precluded the use of perfusion or in situ fixation techniques, it was not unexpected that some fixation artifact would be present. Fixation artifact was most evident in myelin where sloughing and separation were frequently seen (Fig. 4a).

Perhaps the most important finding of this study was the observed electrophysiological changes at a dose of TOCP that did not produce any clinical or histopathological sign of OPIDN. These observations question the validity of the so-called threshold (i.e., 70\% NTE inhibition) necessary for the production of OPIDN. However, since the relationship of the observed electrophysiological changes to the production of OPIDN is not yet known, the idea of a threshold cannot be eliminated. The prevailing hypothesis concerning the mechanism of OPIDN involves the phosphorylation of NTE with subsequent aging of the OP-enzyme complex. The correlation of the electrophysiological changes with aging suggests that the observed axonal conduction deficits are a result of the aging of the OP-enzyme complex. If this is true, it establishes an important link between NTE and clinical OPIDN.

In conclusion, our results show that electrical changes occur in peripheral nerves in response to neuropathogenic OP treatment. OP treatment results in alterations in both relative refractory period and strength-duration curves. In general, these changes are characterized by increased refractoriness in the sci- atic nerve and decreased excitability in both the sciatic and tibial nerves. These abnormalities appear to require both NTE inhibition and aging, rather than inhibition alone. Finally, these changes occurred with TOCP at a dose producing no clinical or histopathological evidence of OPIDN. Future work will deal with the time-course of these electrophysiological deficits, and the effects of pretreatment with nonaging OPs (i.e., DBCV-P) used to block the production of OPIDN.

\section{ACKNOWLEDGMENT}

This work was supported in part by US Army Medical Research and Development Command Contract DAMD-17-C-3187.

\section{REFERENCES}

ABou-DoniA, M. B. (1981). Organophosphorus ester-induced delayed neurotoxicity. Annu. Rev. Pharmacol. Toxicol. 21, 511-548.

ANDERSON, R. J. (1983). Relative refractory period as a measure of peripheral nerve neurotoxicity. Toxicol. Appl. Pharmacol. 71, 391-397.

ANDERSON, R. J., AND DUNHAM, C. B. (1985). Electrophysiologic changes in peripheral nerve following repeated exposure to organophosphorus agents. Arch Toxicol. 58, 97-101.

Anderson, R. J., PARker, J., And Loeb, A. L. (1981). A microprocessor system for controlling stimulation and on-line analysis of electrophysiological parameters. J. Electrophys. Tech. 7, 9-21.

AvERBOOK, B. J., AND ANDERSON, R. J. ( 1983). Electrophysiologic changes associated with chronic administration of organophosphates. Arch. Toxicol 52, 167172.

BAKER, T., AND Lowndes, H. E. (1980). Muscle spindle function in organophosphorus neuropathy. Brain Res. 185, 77-84.

BAKER, T., LOWNDES, H. E, JOHNSON, M. K., AND SANDBORG, I. C. (1980). The effects of phenylmethanesulfonyl fluoride on delayed organophosphorus neuropathy. Arch. Toxicul. 46, 305-311.

DAvis, C. S., JOHNSON, M. K., AND RiCHARDSON, R. J (1985). Organophosphrous compounds. In Neurotoxi cology of Industrial and Commercial Chemicals (J. L. O'Donoghue, Ed.), Vol. II, pp. 1-23. CRC Press, Boca Raton, FL.

Davis, C. S., AND RIChaRdSON, R. J. (1980). Organo- 
phosphorus compounds. In Experimental and Clinicul Neurotoxicology (P. S. Spencer and H. H. Schaumberg, Eds.), pp. 527-544. Williams \& Wilkins, Baltimore.

Dudek, B. R., AND Richardson, R. J. (1980). Lymphocyte and brain neurotoxic esterase: in vitro similarities. Toxicol. Lett. (suppl.), 1, 134.

DUDEK, B. R., AND RICHARDSON, R. J. (1982). Evidence for the existence of neurotoxic esterase in neural and lymphatic tissue of the adult hen. Biochem. Pharmacol. 31, 1117-1121.

Durham, H. D., and Ecobichon, D. J. (1984). The function of motor nerves innervating slow tonic skeletal muscle in hens with delayed neuropathy induced by tri-o-tolyl phosphate. Canad. J. Physiol. Pharmacol. 62, 1268-1273.

EHrich, M. E., JoRTNER, B. S., AND Gross, W. B. (1985). Absence of a protective cffect of corticosterone on 0 -o-diisopropyl phosphorofluoride (DFP) induced delayed neurotoxicity in chickens. Neurotoxicology 6, 87-92.

FRIEDLI, W. G., AND MEYER, M. (1984). Strength-duration curve: A measure for assessing sensory deficit in peripheral neuropathy. J. Neurol. Neurosurg. Psychiatry 47, 184-189.

JOHNSON. M. K. (1974). The primary biochemical lesion leading to the delayed neurotoxic effects of some organophosphorus esters. J. Neurochem. 23, 785-789.

JOHNSON, M. K. (1975). The delayed neuropathy caused by some organophosphorus esters: Mechanism and challenge. CRC Crit. Rev. Toxicol. 3, 289-316.

LOWNDES, H. E., BAKER, T., AND RIKER, W. F. (1974). Motor nerve dysfunction in delayed DFP neuropathy. Eur. J. Pharmacol. 29, 66-73.

RICHARDSON, R. J., AND DUDEK, B. R. (1983). Neurotoxic esterase: Characterization and potential for a predictive screen for exposure to neuropathic organophosphates. In Pesticide Chemistry: Human Welfare and the Environment (J. Miyamoto and P. C. Kearny, Eds.), pp. 491-496. Pergamon, Oxford.

ROBERTS, D. V., AND TROLLOPE, I. E. (1979). Nerve conduction velocity and refractory period as parameters of neurotoxicity. Electroencephalogy. Clin. Neurophysiol. 46, 351-354.

ROBERTSON, D. G., AND ANDERSON, R. J. (1986). Electrophysiological characteristics of hen tibial and sciatic nerve. Amer. J. Vet. Res. 47, 1378-1381.

SCHWAB, B. W., AND RICHARDSON, R. J. (1986). Lymphocyte and brain neurotoxic esterase: Dose and time dependence of inhibition in the hen examined with three organophosphorus esters. Toxicol. Appl. Pharmacol. 83, 1-9. 